\title{
DEPARTAMENTO DE ENFERMAGEM MATERNO-INFANTIL E SAÚDE PÚBLICA DA ESCOLA DE ENFERMAGEM DE RIBEIRÃO PRETO DA UNIVERSIDADE DE SÃO PAULO
}

\author{
Marli Villela Mamede* \\ Maria das Graças Carvalho Ferriani**
}

O Departamento de Enfermagem Materno-Infantil e Saúde Pública e responsável pelas áreas de conhecimento em saúde da criança, saúde da mulher e saúde pública e tem se preocupado em ampliar sua área de conhecimento, expandindo a assistência a mulher para além do ciclo gravídico-puerperal, a assistência à criança em todas as fases de seu crescimento e desenvolvimento, com ênfase especial "a adolescência, e "a assistência a criança hospitalizada. Quanto a saúde do adulto tem buscado incorporar uma visão integral do homem. Na área de saúde pública, também em expansão ampliou-se o conceito do processo saúde-doença na coletividade.

O Departamento é composto por 25 docentes, sendo 2 professores titulares, 03 professores associados, 08 doutores, 10 assistentes e 02 auxiliares de ensino, que compõem as três Áreas de Ensino: Enfermagem Saúde da Criança, Saúde da Mulher e Saúde Publica.

Estes docentes são responsáveis atualmente pela ministração de 1.305 horas didáticas referente ao currículo de graduação em Enfermagem dessa Escola.

O Departamento também participa das atividades de pós-graduação tanto na forma de ministração de disciplinas como orientação de dissertações de mestrado e teses de doutorado dos três programas de pós-graduação dessa Unidade e do Programa de Interunidades do Doutorado das Escolas de Enfermagem da Universidade de São Paulo.

Cabe ressaltar que em 1991 foi criado, neste Departamento, o curso de mestrado em

\footnotetext{
*Professora Doutora e Chefe do Departamento de Enfermagem Materno-Infantil e Saúde Pública da Escola de Enfermagem de Ribeirão Preto da Universidade de Silo Paulo.

**Professora Doutora e Vice-Chefe do Departamento de Enfermagem Materno-Infantil e Saúde Pública da Escola de Enfermagem de Ribeirão Preto da Universidade de São Paulo.
} 
Enfermagem em Saúde Publica, cujo enfoque é direcionado para Enfermagem em Saúde Coletiva, o qual contempla o estudo do processo saúde-doença, desde o enfoque biológico até o social, centrado nos programas de assistência integral a criança, a mulher e ao adulto. Integram ainda esse conhecimento estudos das práticas de saúde e de enfermagem em especial, o sistema e a política de saúde do Brasil. Apresenta uma característica de interdisciplinaridade por contar com a contribuição de disciplinas como Bioestatística, Informática, Nutrição, Ecologia e Epidemiologia.

Em relação às atividades de pesquisa e extensão ressalta-se a criação dos núcleos de estudos, pesquisa e assistência, que através de articulações com a comunidade, instituições de saúde e de educação tem possibilitado consolidar as linhas de pesquisa e o desenvolvimento de importantes serviços integrados, destacando-se, portanto, os seguintes núcleos, de acordo com cada área de ensino:

\section{1. Área Saúde da Criança}

Os docentes da disciplina Enfermagem Pediátrica estão envolvidos em três grandes projetos com a comunidade:

\section{a) Programa de Assistência Primária de Saúde Escolar - "PROASE"}

Objetivos:

- promover a Atenção Integral à Saúde do Escolar, compreendendo ações de promoção, preservação e recuperação da saúde, através da rede de serviços e da ação integrada entre os órgãos dos setores da saúde e da educação;

- contribuir para melhoria de saúde e, conseqüentemente, do processo de desenvolvimento e formação integral da criança;

- analisar o processo de trabalho em saúde na assistência ao escolar, entendendo-se não como uma faixa etária, mas como uma assistência a saúde mediada pelo setor de Educação abrangendo do pré-escolar ao adolescente.

O PROASE encontra-se implantado em todas as escolas de $1^{\circ} \mathrm{grau}$ da rede pública de Ribeirão Preto, atendendo cerca de 75 mil escolares.

Este trabalho, é resultado de um Convênio entre a Escola de Enfermagem de Ribeirão Preto e a Prefeitura Municipal de Ribeirão Preto tendo como interveniente as Secretarias Municipais da Saúde e da Educação.

O PROASE é coordenado por dois docentes da disciplina de Enfermagem Pediátrica desde de 1980 e é desenvolvido por uma equipe multidisciplinar (enfermeiros, pediatras, psicólogo, fonoaudiólogo, oftalmologistas, otorrino, laringologistas, dentre outros).

A contra partida da Prefeitura Municipal de Ribeirão Preto e contratação de recursos humanos e manutenção de recursos materiais para o desenvolvimento do projeto na rede.

b) Grupo de Estudos sobre Saúde da Criança e do Adolescente - "GESCA" Objetivos:

- promover estudos e pesquisas sobre o processo saúde-doença e a assistência a saúde da 
criança, apreendendo-a em suas relações com a família, escola, o setor saúde e em suas necessidades no processo de crescimento e desenvolvimento;

- apreender a incorporação da enfermagem nas diferentes modalidades da prática médica e das práticas em saúde na assistência à criança e programas de ensino de enfermagem pediátrica nível de graduação e extensão universitária.

O GESCA está aberto ao intercâmbio com outras instituições; no momento, participam docentes da Universidade Federal de São Carlos, da Faculdade de Enfermagem de Botucatú da Universidade Estadual Paulista, enfermeiros da Secretaria Municipal da Saúde, entre outros. Quanto as fontes de recursos este está aguardando parecer do Conselho Nacional de Desenvolvimento Cientifico e Tecnológico (CNPq) para o financiamento do projeto. Este projeto de extensão há participação dos 7 docentes da área.

\section{c) Grupo de Apoio á Criança com Câncer - "GACC"}

Objetivos:

- procura facilitar a criança e a sua família a convivência com o câncer, convivência esta sabidamente sofrida e desgastante.

Este grupo é composto por médicos, enfermeiras, assistentes sociais, psicólogas e nutricionistas do Hospital das Clínicas da Faculdade de Medicina de Ribeirão Preto da Universidade de São Paulo, Departamento de Puericultura e Pediatria da Universidade de São Paulo e Departamentos de Enfermagem Materno-Infantil e Saúde Pública e Enfermagem psiquiátrica e Ciências Humanas da Escola de Enfermagem de Ribeirão Preto da Universidade de São Paulo.

O grupo vem trabalhando com as questões do câncer infantil e desenvolvendo atividades técnico-científica e assistenciais. Há participação neste trabalho de 2 docentes da área de enfermagem pediátrica.

Com relação a recursos, estes são da própria comunidade uma vez que existe um grupo de voluntárias responsável pela aquisição de fundos para a manutenção das atividades do grupo, entre as quais destaca-se a Casa de Apoio à Criança com Câncer, situada neste Campus.

\section{2. Área Saúde da Mulher}

Os docentes envolvidos nessa área estão engajados nos seguintes projetos com a comunidade:

\section{a) Núcleo de Aleitamento Materno - "NALMA"}

Objetivos:

- prestar assistência principal mente ao binômio mãe-filho, valendo-se de uma abordagem humanístico-cientítica;

- preparar profissionais que atuam na assistência a puérpera;

- promover cursos, seminários e atividades afins para se debater a questão em pauta;

- realizar intercâmbio com órgãos nacionais e internacionais que trabalham com 
amamentação;

- criar mecanismos de articulação inter-institucionais e inter-profissionais no sentido de promover e incentivar o aleitamento materno;

- implementar pesquisas sobre aleitamento materno.

Prioriza o treinamento dos profissionais de saúde das Unidades Básicas de Saúde de Ribeirão Preto para adquirirem competência técnica e cientifica a fim de trabalharem as questões pertinentes ao aleitamento materno. Atuam como agentes multiplicadores de conhecimento nesta área.

\section{b) Núcleo de Ensino, Assistência e Pesquisa em Reabilitação de mastectomiazadas - "REMA"}

Objetivos:

- prestar assistência integral a mulher com câncer mamário e seus familiares, estimulando a busca de um maior desempenho de seus papeis e habilidades dentro de seu contexto social;

- incrementar a formação de uma equipe multidisciplinar para assistência integral da mulher com câncer de mama;

- auxiliar no treinamento e capacitação de profissionais, alunos de graduação e pósgraduação em enfermagem e outras áreas de saúde, para assistência integral a mastectomizadas;

- prestar assessoria a outras instituições de saúde na assistência integral a mastectomizadas;

- desenvolver programas de prevenção e detecção precoce do câncer ginecológico e mamário;

- desenvolver pesquisas sobre o câncer ginecológico e mamário.

Este núcleo, funciona na própria Escola de Enfermagem de Ribeirão Preto, em um Laboratório apropriado para atender as necessidades da população alvo. O atendimento ocorre todas as segundas, quartas e sextas-feiras, das 8:00 às 12:00 horas. Este trabalho e desenvolvido por 3 docentes da área da saúde da mulher, uma enfermeira e técnica especializada do Departamento de Enfermagem Materno-Infantil e Saúde Publica e há o envolvimento de mais 2 docentes dos outros Departamentos da Escola de Enfermagem de Ribeirão Preto da Universidade de São Paulo (Enfermagem Geral e Especializada e Enfermagem psiquiátrica e Ciências Humanas). Há participação de enfermeiras bolsistas de Aperfeiçoamento - tipo pesquisa do CNPq, pós-graduandas e de uma fisioterapeuta estagiaria.

O REMA teve início em 1989 e desde da sua criação, vem sendo reconhecido pela Secretaria do Estado de São Paulo, quanto a necessidade de multiplicar o trabalho. Diante dessa necessidade, vem sendo oferecido curso de aperfeiçoamento, com carga horária de 180 horas, para os profissionais da referida Secretaria objetivando instrumentalizar a nível teórico e prático, uma equipe multiprofissional.

\section{c) Pesquisa Transcultural na Área Saúde da Mulher:}

- objetiva estudar e identificar os problemas de saúde vivenciados pelas mulheres de diferentes culturas, regiões e países, em especial nas situações de ameaça a sua sexualidade.

Este projeto faz parte de um convênio nacional assinado entre a Escola de Enfermagem 
de Ribeirão Preto da Universidade de São Paulo com a Universidade Federal do Ceará Fortaleza e a nível internacional com a University of Illinois at Chicago.

Para o desenvolvimento deste projeto a nível nacional, o Departamento de Enfermagem Materno-Infantil e Saúde Pública conta com a participação de docentes da Escola de Enfermagem de Ribeirão Preto da Universidade de São Paulo, do Departamento de Enfermagem da UNICAMP e da Universidade Federal do Ceará.

\section{3. Área de Saúde Pública}

Os docentes envolvidos nessa área encontram-se realizando os seguintes projetos:

\section{a) Núcleo de pesquisa e Estudos em Saúde Coletiva - "NUPESCO"}

Objetivos:

- constituir um grupo de pesquisadores com formação em saúde coletiva (com ênfase na enfermagem) contribuindo para a reorganização dos serviços de saúde e a melhoria de enfermagem.

O Núcleo se caracteriza como um grupo de estudo, mas não deixa também de apresentar aderência a área de extensão, haja vista estar iniciando neste ano de 1993 um projeto, junto a Secretaria Municipal de Saúde sobre Gerenciamento de Serviços a nível da rede básica de saúde, envolvendo as questões do ensino de saúde pública e a pesquisa em saúde coletiva.

Esta aderência à prática que caracteriza os seus aspectos de extensão pode ser observada através de sua produção científica, que em sua grande maioria, contempla os serviços de saúde, mais especificamente o trabalho de enfermagem.

O Núcleo compõe-se de docentes do Departamento de Enfermagem Materno-Infantil e Saúde Pública, Departamento de Enfermagem Geral e Especializada, de outras instituições de ensino, bem como de enfermeiros de serviço.

\section{b) Núcleo de AIDS - Doenças Sexualmente Transmissíveis - "NAIDST"}

Objetivos:

- formar e capacitar profissionais da área da saúde para assistência a pacientes portadores de AIDS e DST;

- promover programas informativos e educativos para o público em geral;

- implementar pesquisa em AIDS e DST.

Este trabalho é realizado por docentes dos três Departamentos da Escola de Enfermagem de Ribeirão Preto da Universidade de São Paulo (MISP, EGE e EPCH).

O projeto de Controle e Prevenção da AIDS na cidade de Ribeirão Preto, envolve um trabalho educativo a todos os segmentos da sociedade (criança, adolescentes, adultos, idosos) através de cursos, palestras, reciclagem, treinamento de profissionais da área da saúde.

Conta com a colaboração do Hospital das Clínicas, SUS e Secretaria Municipal.

\section{c) Grupo de Recursos Humanos em Enfermagem}

Objetivo:

- estudar a questão dos Recursos Humanos em Enfermagem em todos os âmbitos desde a 
formação até a utilização desse pessoal, em todos os níveis, pelo mercado de trabalho.

A meta do grupo é uma maior integração com o pessoal de enfermagem das áreas de ensino e dos serviços. O mesmo pode ser dito em relação às esferas centrais, como o Ministério da Saúde.

Este trabalho é desenvolvido por docentes da área de saúde pública do Departamento de Enfermagem Materno-Infantil e Saúde Pública e do Departamento de Enfermagem Geral e Especializada.

d) Núcleo de Estudos sobre Saúde e Trabalho - "NUESAT"

Objetivos:

A pesquisa, o ensino e a extensão dos serviços à comunidade, na área saúde e trabalho.

Este núcleo é formado por docentes dos três Departamentos da Escola de Enfermagem de Ribeirão Preto da Universidade de São Paulo, docente da Universidade Federal de São Carlos, Membros da Sub-Delegacia Regional do Trabalho e enfermeiros do trabalho, que atuam na região. 